\title{
Nonstatistical UV Fragmentation of Gas-Phase Peptides Reveals Conformers and Their Structural Features
}

\author{
Vladimir Kopysov, ${ }^{1}$ Alexander Makarov $^{2} \&$ Oleg V. Boyarkin ${ }^{1 *}$ \\ ${ }^{1}$ Laboratoire de Chimie Physique Moléculaire, École Polytechnique Fédérale de Lausanne, CH-1015 \\ Lausanne, Switzerland. \\ ${ }^{2}$ Thermo Fisher Scientific, Hanna-Kunath Str. 11, 28199 Bremen, Germany. \\ *Correspondence should be addressed to O.V.B. (oleg.boiarkin@epfl.ch).
}

\section{Contents}

1. Experimental details and data analysis

1.1. UV spectroscopy and mass spectrometry of cold ions $\quad$ S2

1.2. Data acquisition and processing $\quad$ S2

$\begin{array}{ll}\text { 1.3. Matrix factorization } & \mathrm{S} 4\end{array}$

$\begin{array}{ll}\text { 1.4. Bi-cross-validation } & \mathrm{S} 4\end{array}$

$\begin{array}{ll}\text { 1.5. Contribution of individual components } & \text { S7 }\end{array}$

2. 2D UV-MS spectra

2.1. Figure S1. 2D UV-MS spectrum of singly protonated Tyr-Ala dipeptide $\quad$ S8

2.2. Figure S2. 2D UV-MS spectrum of doubly protonated gramicidin S $\quad$ S9

2.3. Figure S3. Plot of BCV error for 2D UV-MS spectrum of doubly protonated gramicidin S $\quad$ S10

3. Details of computations $\quad$ S11

4. Results of calculations for the four lowest-energy conformers of singly protonated Tyr-Ala dipeptide

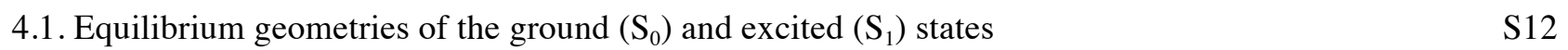

4.2. Zero-point vibrational energies in the ground $\left(\mathrm{S}_{0}\right)$ and excited $\left(\mathrm{S}_{1}\right)$ states $\quad \mathrm{S} 16$

$\begin{array}{ll}\text { 4.3. Figure S4. Simulated shape of the UV band origins at } \mathrm{m} / \mathrm{z}=137.080 \mathrm{Da} & \mathrm{S} 18\end{array}$

$\begin{array}{ll}\text { References } & \text { S19 }\end{array}$ 


\subsection{UV spectroscopy and mass spectrometry of cold ions}

Protonated peptides are generated from the solutions using a nanoelectrospray ion source and are guided into the vacuum chamber through a $0.7 \mathrm{~mm}$ ID $90 \mathrm{~mm}$ long stainless steel capillary and orthogonally injected into an electrodynamic ion funnel that works at a pressure of $\sim 10 \mathrm{mbar}$. The ions are then guided through another ion funnel held at a pressure of $\sim 1.5$ mbar and accumulated (for $\sim 200-500 \mathrm{~ms}$ ) in a hexapole ion trap. The trapped ions are then released from the hexapole and transmitted through a low-resolution $(\sim 100)$ quadrupole mass filter, which selects parent ions of a particular mass-to-charge ratio (m/z). An RF-only octopole guides them into an octopole ion trap, ${ }^{1}$ which is cooled to $6 \mathrm{~K}$ by a closed-cycle refrigerator (SRDK-408, Sumitomo). Ions in the trap are cooled in collisions with a pulse of helium atoms, which are introduced into the trap before the arrival of the ion packet. Approximately $40 \mathrm{~ms}$ later, when ions are cooled and helium has been pumped out, we interrogate the ions in the trap with a UV laser pulse. Shortly after this, the content of the cold trap is released and the fragment and parent ions are guided into the Exactive Orbitrapbased mass spectrometer (Thermo Fisher Scientific, Bremen, Germany) for mass analysis. The cold ion spectrometer (CIS) is coupled to the Exactive MS by replacing the original ion source section of the MS with a two-section octopole ion guide. The duty cycles of the laser and cold ion spectrometer (typically 10 or $20 \mathrm{~Hz}$ ) are synchronized with the Orbitrap analyzer by triggering a master pulse generator of CIS using a small fraction of the electrical pulse, applied to the entrance split lens of the Exactive MS. The computer-controlled data acquisition system, which is synchronized with CIS records UV laser power, measured by a pyroelectric detector, and the laser wavenumber, measured by a wavelength meter (HF-Angstrom WS/5, $\pm 0.1 \mathrm{~cm}^{-1}$ accuracy).

UV photofragmentation is performed using 2-3 $\mathrm{mJ}$ output of a frequency-doubled (in a type-II BBO crystal) dye laser (Lumonics HD500) pumped by 7 ns pulses of a Nd:YAG laser (Spectra Physics GCR-210), configured to generate its third harmonic $(355 \mathrm{~nm})$. The UV laser beam enters and exits the vacuum chamber through $\mathrm{BaF}_{2}$ windows and is loosely focused by a fused silica lens of $F=75 \mathrm{~cm}$ onto the trap axis $\sim 3 \mathrm{~cm}$ outside of its entrance to ensure an overlap of the whole ion cloud in the trap by the UV beam. We use Coumarin-500 and Coumarin 540A laser dyes (Exciton) to cover the spectral range of $260-285 \mathrm{~nm}$. The laser linewidth is $0.2 \mathrm{~cm}^{-1}$.

\subsection{Data acquisition and processing}

At each UV wavenumber we measure a single mass spectrum (MS) by averaging 2-10 microscans of the Orbitrap analyzer, such that each microscan corresponds to a single laser shot. Time synchronization of the Orbitrap and the laser allows the data acquisition software to label each mass spectrum by the precisely measured wavenumber and to record the UV laser pulse energy in 
the respective cycles of measurements. We then normalize each mass spectrum to the recorded laser pulse energy.

We measure 2D UV-MS spectrum by continuously recording the mass spectra on the Orbitrap while scanning the UV laser. Therefore, the produced data is a set of fragment mass spectra stored as a Thermo RAW file and a set of pairs of wavenumber and UV pulse energy stored as a text file. In the recorded spectrum the spectral resolution is $0.2 \mathrm{~cm}^{-1}$ and the mass resolving power is $10^{4}-10^{5}$.

The recorded mass spectra are first converted from the RAW data format into a text file using the Thermo Scientific MSFileReader library. The whole $\mathrm{m} / \mathrm{z}$ range is, first, divided into a set of equal bins of 0.1 Da size. Each high-resolution mass scan from the RAW file is then sampled over this set and the resulting array of ion intensities is written as a single space-separated string in a text file. Thus, the output text file contains the recorded 2D spectrum in a matrix form, where the rows correspond to the different laser wavenumbers and the columns correspond to the different $\mathrm{m} / \mathrm{z}$ values. This matrix is then loaded and processed in MATLAB software suite.

As the first step of the data matrix processing, the 2D spectrum is to be baseline-corrected using any suitable approach developed for mass spectrometry. We perform this step using our own approach as described below. Each column of the matrix represents the UV spectrum recorded at a certain $\mathrm{m} / \mathrm{z}$ value. The spectrum is smoothed using a Savitzky-Golay filter ${ }^{2}$ (5-point window, quadratic polynomial). The sum of such a smoothed spectrum over the whole wavenumber range gives an estimated "signal" value and the norm of the residual between the smoothed and raw spectrum gives an estimated "noise" value. By calculating the signal and noise for all the $\mathrm{m} / \mathrm{z}$ values, we obtain an integrated over wavenumber mass spectrum. Typically, a UV fragmentation spectrum contains only a few peaks of an appreciable intensity, but large peak-free regions. The second derivative of the integrated mass spectrum is used to find these regions. Two types of points are considered to be peak-related: positive local maxima and those negative local minima, which absolute values are below some threshold. The choice of the threshold strikes a compromise between the loss of some peaks (for a too high threshold) and an erroneous assignment of the noise spikes as truly mass peaks (for a too low threshold). We chose the threshold of 20 for the signal-tonoise ratio as this compromise. In addition to this, we remove the peaks that systematically appear as artifacts in our mass spectra. After the identification of the peaks-free regions, for each mass spectrum we employ the shape-preserving piecewise cubic interpolation, taking these regions as a set of sample data points. This gives us an estimate of the baseline position. By subtracting it from the raw mass spectra we obtain the baseline-corrected mass spectra. All the negative values in the resulted $2 \mathrm{D}$ spectrum are replaced by zeros. Finally, we transform the baseline-corrected 2D spectrum into a matrix, which represents a set of UV spectra measured at the most prominent fragment and parent mass peaks. 


\subsection{Matrix factorization}

The preprocessed matrix $\boldsymbol{D}$ is factorized into a non-negative matrix $\boldsymbol{W}$ of UV spectra and a nonnegative matrix $\boldsymbol{H}$ of MS spectra of different components. If $\boldsymbol{k}$ denotes the number of such components, then the $\boldsymbol{n}$-by- $\boldsymbol{m}$ matrix $\boldsymbol{D}$ can be factorized into $\boldsymbol{n}$-by- $\boldsymbol{k}$ matrix $\boldsymbol{W}$ and $\boldsymbol{k}$-by- $\boldsymbol{m}$ matrix $\boldsymbol{H}$, so that their matrix product $\boldsymbol{W H}$ is a lower-rank approximation to $\boldsymbol{D}^{3}{ }^{3}$ Since all the matrices, $\boldsymbol{D}$, $\boldsymbol{W}$ and $\boldsymbol{H}$, are non-negative, this approach was called non-negative matrix factorization (NMF). ${ }^{4}$ One can find the best $\boldsymbol{k}$-rank approximation by minimizing the Frobenius norm of the residual:

$$
\|D-W \cdot H\|_{F}=\sqrt{\sum_{i=1}^{n} \sum_{j=1}^{m}\left(D_{i j}-\sum_{l=1}^{k} W_{i l} \cdot H_{l j}\right)^{2}}
$$

For this we use the MATLAB built-in function $n n m f$, which employs the iterative alternating least-squares algorithm. The maximum number of iterations for each factorization procedure was 1000. In order to find the global solution, we have repeated the factorization of the UV-MS matrix 100 times for each rank $\boldsymbol{k}$ from 1 to 5 . The accuracy of the NMF can be assessed as the root-meansquare deviation (RMSD) between the matrix $\boldsymbol{D}$ and its $\boldsymbol{k}$-rank approximation:

$$
\operatorname{RMSD}(k)=\frac{\left\|D^{n \times m}-W^{n \times k} \cdot H^{k \times m}\right\|_{F}}{\sqrt{n \cdot m}}
$$

where superscripts denote the size of the matrices. Instead of absolute RMSD values one can also use the relative RMSD:

$$
R M S D_{\text {rel }}(k)=\frac{R M S D(k)}{R M S D(0)}=\frac{\left\|D^{n \times m}-W^{n \times k} \cdot H^{k \times m}\right\|_{F}}{\left\|D^{n \times m}\right\|_{F}}
$$

However, the choice of the number of significant components, which correspond to the conformers rather than to the noise in experimental data, is problematic, since larger values of $\boldsymbol{k}$ always result in a better approximation to $\boldsymbol{D}$ and hence to lower RMSD (see Figure S3). Here to estimate the number of important components we employed another approach, which is called bicross-validation $(\mathrm{BCV}){ }^{5}$

\subsection{Bi-cross-validation}

The general idea behind the BCV is to hold out a submatrix of the matrix $\boldsymbol{D}$ (i.e., a set of elements of $\boldsymbol{D}$ ) and use the rest of $\boldsymbol{D}$ to predict the held out submatrix. The quality of the $\boldsymbol{k}$-term NMF is expressed by a BCV error. For instance, in the case of $\boldsymbol{r}$-by-s submatrices ( $\boldsymbol{r}$ and $\boldsymbol{s}$ are such small integers, that $\boldsymbol{n}$ and $\boldsymbol{m}$ are the multiples of $\boldsymbol{r}$ and $\boldsymbol{s}$, respectively) the BCV error is evaluated as follows. The matrix $\boldsymbol{D}$ is partitioned into $(u \times v)$ submatrices $(u=n / r, v=m / s)$ : 


$$
D^{n \times m}=\left(\begin{array}{ccc}
D_{11}^{r \times s} & \cdots & D_{1 v}^{r \times s} \\
\vdots & \ddots & \vdots \\
D_{u 1}^{r \times s} & \cdots & D_{u v}^{r \times s}
\end{array}\right)
$$

Then, for a given $i \in\{1, \ldots, u\}$ and $j \in\{1, \ldots, v\}$, the matrix $\boldsymbol{D}$ has the form

$$
D^{n \times m}=\left(\begin{array}{ccc}
X_{11}^{(i-1) r \times(j-1) s} & Z_{1}^{(i-1) r \times s} & X_{12}^{(i-1) r \times(v-j) s} \\
Y_{1}^{r \times(j-1) s} & D_{i j}^{r \times s} & Y_{2}^{r \times(v-j) s} \\
X_{21}^{(u-i) r \times(j-1) s} & Z_{2}^{(u-i) r \times s} & X_{22}^{(u-i) r \times(v-j) s}
\end{array}\right)
$$

Any permutation of rows and columns of $\boldsymbol{D}$ results in the corresponding permutations of rows of $\boldsymbol{W}$ and columns of $\boldsymbol{H}$, respectively, but, in general, does not affect the NMF (in terms of RMSD). Therefore, one can rearrange $\boldsymbol{D}$ in such a way that $\boldsymbol{D}_{i j}$ will be the upper left submatrix

$$
\left(\begin{array}{ccc}
D_{i j}^{r \times s} & Y_{1}^{r \times(j-1) s} & Y_{2}^{r \times(v-j) s} \\
Z_{1}^{(i-1) r \times s} & X_{11}^{(i-1) r \times(j-1) s} & X_{12}^{(i-1) r \times(v-j) s} \\
Z_{2}^{(u-i) r \times s} & X_{21}^{(u-i) r \times(j-1) s} & X_{22}^{(u-i) r \times(v-j) s}
\end{array}\right)=\left(\begin{array}{cc}
D_{i j}^{r \times s} & Y^{r \times(m-s)} \\
Z^{(n-r) \times s} & X^{(n-r) \times(m-s)}
\end{array}\right)
$$

where the submatrices $\boldsymbol{X}, \boldsymbol{Y}$ and $\boldsymbol{Z}$ are defined as

$$
\begin{aligned}
& X^{(n-r) \times(m-s)}=\left(\begin{array}{ll}
X_{11}^{(i-1) r \times(j-1) s} & X_{12}^{(i-1) r \times(v-j) s} \\
X_{21}^{(u-i) r \times(j-1) s} & X_{22}^{(u-i) r \times(v-j) s}
\end{array}\right) \\
& Y^{r \times(m-s)}=\left(\begin{array}{ll}
Y_{1}^{r \times(j-1) s} & Y_{2}^{r \times(v-j) s}
\end{array}\right) \\
& Z^{(n-r) \times s}=\left(\begin{array}{l}
Z_{1}^{(i-1) r \times s} \\
Z_{2}^{(u-i) r \times s}
\end{array}\right)
\end{aligned}
$$

Since both $\boldsymbol{r}$ and $\boldsymbol{s}$ are small, the submatrix $\boldsymbol{X}$ contains almost the same information as the entire matrix $\boldsymbol{D}$ and the $\boldsymbol{k}$-term NMF of $\boldsymbol{X}$ is likely to be of the same quality as that of $\boldsymbol{D}$. Literally, this means that, if the transformed matrix $\boldsymbol{D}$ (in which $\boldsymbol{D}_{i j}$ is the upper left submatrix) is factorized into $\boldsymbol{W}$ and $\boldsymbol{H}$, so that

$$
\left(\begin{array}{cc}
D_{i j}^{r \times s} & Y^{r \times(m-s)} \\
Z^{(n-r) \times s} & X^{(n-r) \times(m-s)}
\end{array}\right)=D^{n \times m} \approx W^{n \times k} \cdot H^{k \times m}=\left(\begin{array}{c}
\widehat{W}_{D}^{r \times k} \\
\widehat{W}_{X}^{(n-r) \times k}
\end{array}\right) \cdot\left(\begin{array}{ll}
\widehat{H}_{D}^{k \times s} & \widehat{H}_{X}^{k \times(m-s)}
\end{array}\right)
$$

then the $\boldsymbol{k}$-term NMF of $\boldsymbol{X}$ itself can be approximated as

$$
X^{(n-r) \times(m-s)} \approx W_{X}^{(n-r) \times k} \cdot H_{X}^{k \times(m-s)} \approx \widehat{W}_{X}^{(n-r) \times k} \cdot \widehat{H}_{X}^{k \times(m-s)}
$$

Within the accuracy of the NMF of the entire matrix $\boldsymbol{D}$, the approximation to $\boldsymbol{D}_{i j}$ is

$$
D_{i j}^{r \times s} \approx \widehat{D}_{i j}^{r \times s}=\widehat{W}_{D}^{r \times k} \cdot \widehat{H}_{D}^{k \times s}
$$

and using the following two approximations

$$
\begin{aligned}
& \widehat{W}_{D}^{r \times k} \approx \widehat{W}_{D}^{r \times k} \cdot \widehat{H}_{X}^{k \times(m-s)} \cdot\left(\widehat{H}_{X}^{k \times(m-s)}\right)^{+} \approx Y^{r \times(m-s)} \cdot\left(H_{X}^{k \times(m-s)}\right)^{+} \\
& \widehat{H}_{D}^{k \times s} \approx\left(\widehat{W}_{X}^{(n-r) \times k}\right)^{+} \cdot \widehat{W}_{X}^{(n-r) \times k} \cdot \widehat{H}_{D}^{k \times s} \approx\left(W_{X}^{(n-r) \times k}\right)^{+} \cdot Z^{(n-r) \times s}
\end{aligned}
$$

one can finally get 


$$
\begin{gathered}
D_{i j}^{r \times s} \approx \widehat{D}_{i j}^{r \times s} \approx Y^{r \times(m-s)} \cdot\left(H_{X}^{k \times(m-s)}\right)^{+} \cdot\left(W_{X}^{(n-r) \times k}\right)^{+} \cdot Z^{(n-r) \times s} \approx \\
\approx Y^{r \times(m-s)} \cdot\left(W_{X}^{(n-r) \times k} \cdot H_{X}^{k \times(m-s)}\right)^{+} \cdot Z^{(n-r) \times s} \approx Y^{r \times(m-s)} \cdot\left(X^{(n-r) \times(m-s)}\right)^{+} \cdot Z^{(n-r) \times s}
\end{gathered}
$$

where the superscript "+" denotes matrix pseudoinversion. In fact, this means, that the small submatrix $\boldsymbol{D}_{i j}$ can be reconstructed on the basis of the other three submatrices:

$$
D_{i j}^{r \times s} \approx Y^{r \times(m-s)} \cdot\left(X^{(n-r) \times(m-s)}\right)^{+} \cdot Z^{(n-r) \times s}
$$

Furthermore, this approximation becomes the exact equality, provided the rank of the submatrix $\boldsymbol{X}$ equals the rank of the entire matrix $\boldsymbol{D}$, that is,

$$
D_{i j}^{r \times s}=Y^{r \times(m-s)} \cdot\left(X^{(n-r) \times(m-s)}\right)^{+} \cdot Z^{(n-r) \times s}
$$

The accuracy of the approximation of $\boldsymbol{D}_{i j}$ is assessed by means of Frobenius norm of the residual

$$
\left\|D_{i j}^{r \times s}-\widehat{D}_{i j}^{r \times s}\right\|_{F}
$$

Since the procedure, described above, can be performed for any $i \in\{1, \ldots, u\}$ and $j \in\{1, \ldots, v\}$, the $\mathrm{BCV}$ error is defined as

$$
B C V(k)=\sqrt{\frac{\sum_{i=1}^{u} \sum_{j=1}^{v}\left\|D_{i j}^{r \times s}-\widehat{D}_{i j}^{r \times s}\right\|_{F}^{2}}{n \cdot m}}
$$

As in the case of RMSD, one may also use the relative BCV error:

$$
B C V_{r e l}(k)=\frac{B C V(k)}{B C V(0)}=\frac{\sqrt{\sum_{i=1}^{u} \sum_{j=1}^{v}\left\|D_{i j}^{r \times s}-\widehat{D}_{i j}^{r \times s}\right\|_{F}^{2}}}{\left\|D^{n \times m}\right\|_{F}}
$$

When the value of $\boldsymbol{k}$ is less than the true number of components, the BCV error is mainly determined by the NMF itself (low accuracy of the $\boldsymbol{k}$-rank approximation), and therefore BCV error is close to the RMSD error. When the value of $\boldsymbol{k}$ becomes greater than the true number of components, the NMF starts to fit the noise in the experimental data by the redundant components, which is called overfitting. However, we use the NMF of the submatrix $\boldsymbol{X}$ to estimate the submatrix $\boldsymbol{D}_{i j}$. The overfitting of $\boldsymbol{X}$ does not lead to a better approximation to $\boldsymbol{D}_{i j}$, because the noise is not correlated in these two submatrices. Thus, the large values of $\boldsymbol{k}$ will not yield a smaller BCV error, while the RMSD error monotonically decreases upon increasing $\boldsymbol{k}$ (see Figure S3).

\subsection{Contribution of individual components}

One can also estimate the importance of the obtained components by comparing their relative contributions to the decomposition of the initial matrix $\boldsymbol{D}$ in the following way. The columns of the matrix $\boldsymbol{W}$ and the rows of the matrix $\boldsymbol{H}$ represent the UV and MS vectors of the components, respectively. The outer product of each pair of vectors that correspond to UV and MS spectra of a 
certain component gives the UV-MS matrix, so that the matrix $\boldsymbol{D}$ is a sum of UV-MS matrices of the retained components:

$$
D \approx \sum_{l=1}^{k} D_{l}=\sum_{l=1}^{k} W_{\bullet l} \otimes H_{l}
$$

The sum over all the elements of the matrix $\boldsymbol{D}$ is a measure of the integral fragmentation efficiency (over the whole range of the wavenumbers and all the fragments) of all the ionic species in a sample and the identical sum for the matrix $\boldsymbol{D}_{\boldsymbol{l}}$ is the photofragmentation efficiency of the $\boldsymbol{l}$-th component. Thus, we can derive the following quantity that characterizes the relative contribution of the $\boldsymbol{l}$-th component:

$$
C_{l}=100 \% \cdot \frac{\sum_{i, j}\left(D_{l}\right)_{i j}}{\sum_{p=1}^{k} \sum_{i, j}\left(D_{p}\right)_{i j}}
$$

where $\left(\boldsymbol{D}_{l}\right)_{i j}$ is the element of the matrix $\boldsymbol{D}_{\boldsymbol{l}}$ from the $\boldsymbol{i}$-th row and $\boldsymbol{j}$-th column. The contribution $\boldsymbol{D}_{\boldsymbol{l}}$ reflects the relative concentration of the $\boldsymbol{l}$-th component in a sample as well as its absorption crosssection and fragmentation efficiency. 


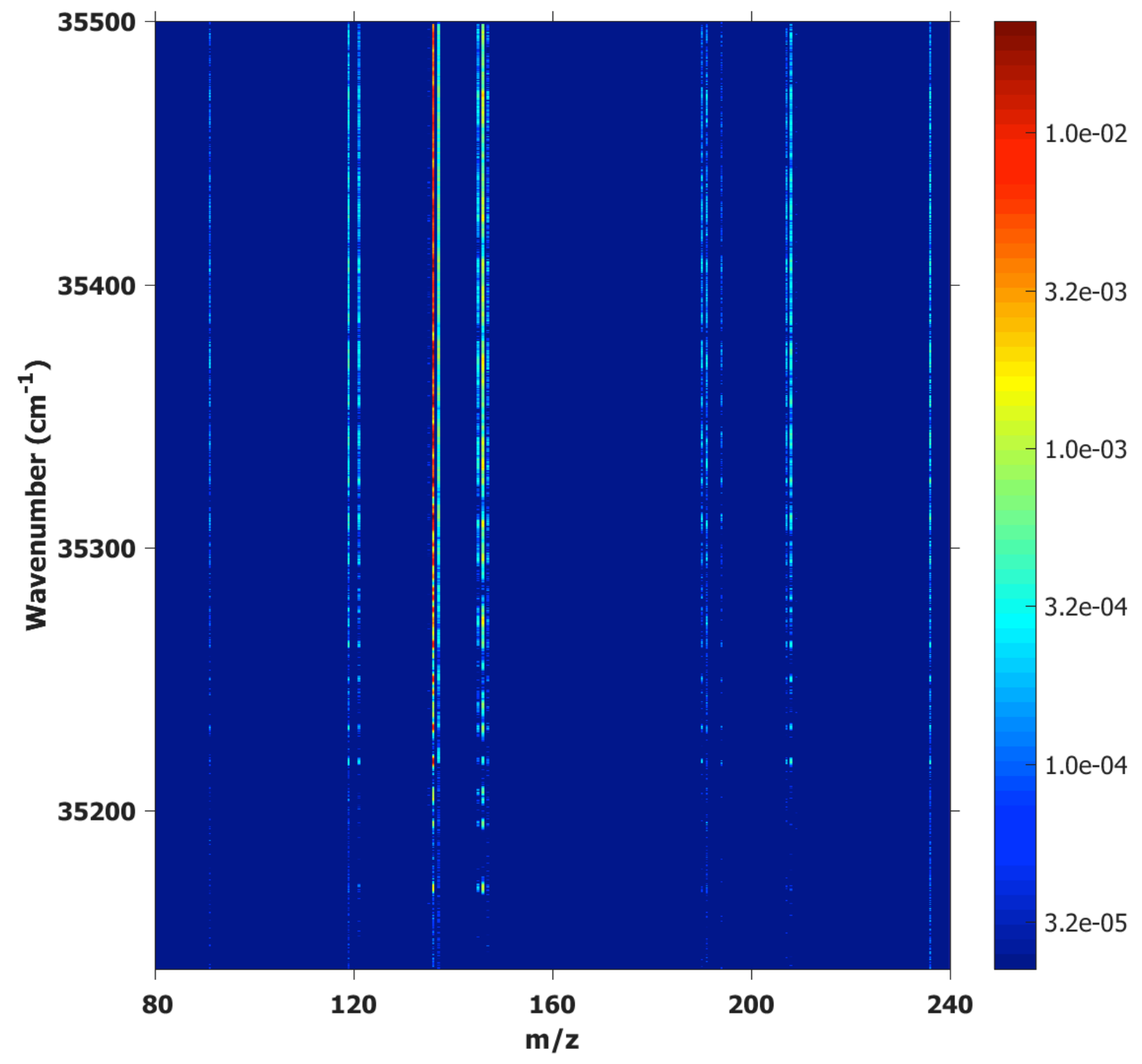

Figure S1. 2D UV-MS spectrum of singly protonated Tyr-Ala dipeptide. The traces are artificially broadened on the $\mathrm{m} / \mathrm{z}$ scale for graphical clarity. 


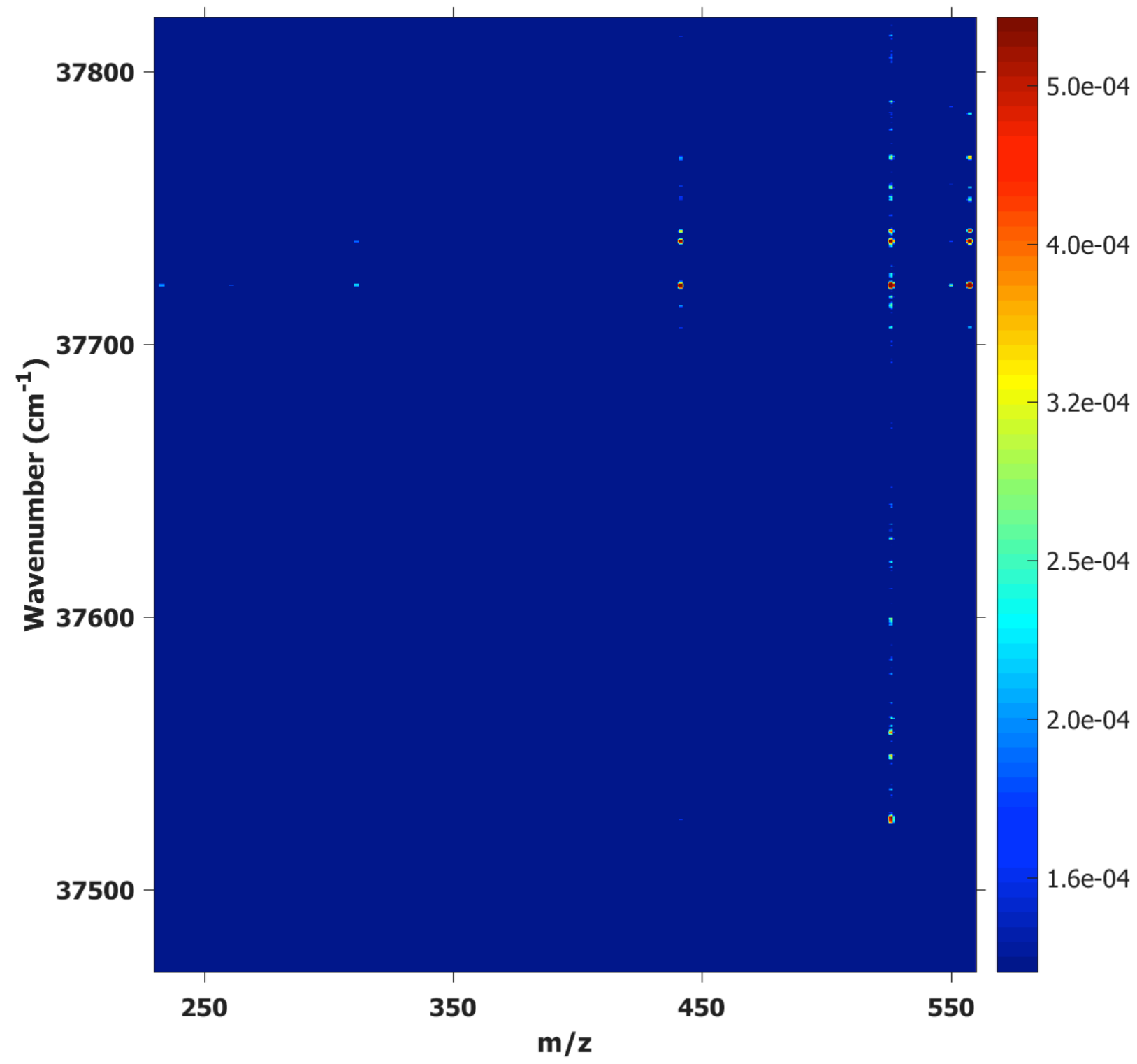

Figure S2. 2D UV-MS spectrum of doubly protonated gramicidin S. The traces are artificially broadened on the $\mathrm{m} / \mathrm{z}$ scale for graphical clarity. 


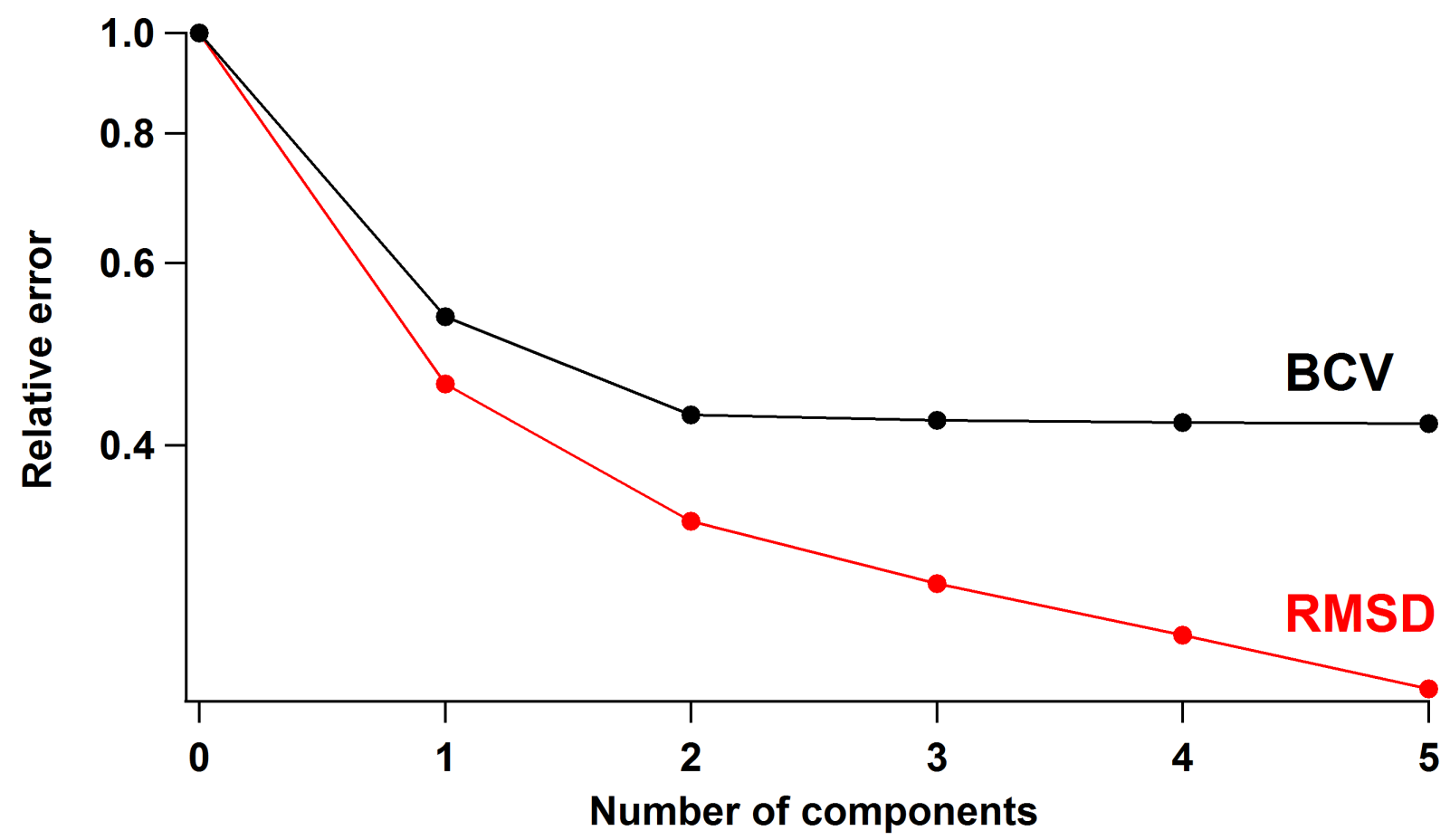

Figure S3. Plot of RMSD and BCV relative errors as functions of the number of components, $\boldsymbol{k}$, for 2D UV-MS spectrum of doubly protonated gramicidin S. The BCV error converges at $\boldsymbol{k}=\mathbf{2}$, while the RMSD error monotonically decreases. For details see Section 1.4. 


\section{Details of computations}

In order to evaluate the spectral shifts of the band origins of the conformers of $[\mathrm{YA}+\mathrm{H}]^{+}$dipeptide upon their isotopic ${ }^{12} \mathrm{C}-{ }^{13} \mathrm{C}$ substitution we took the coordinates of the four lowest-energy structures, calculated in ref 6 . The geometry of the conformers with the relative energy of $0.000,0.046,0.844$ and $0.896 \mathrm{kcal} / \mathrm{mol}$ are in a good agreement with those calculated by Stearns et al. ${ }^{7}$ The first two structures correspond to the conformers A and C (component II from our analysis), while the other two - to conformers B and D (component I from our analysis).

Ground-state geometry of all four conformers was re-optimized and subsequently used as a starting point for the $S_{1}$ excited state geometry optimization. Both ground-state and excited-state geometry optimizations were performed with the convergence criterion of $10^{-5}$ a.u. for the atomic forces. Then the Hessian matrices were calculated numerically for $S_{0}$ and $S_{1}$ equilibrium geometries of each conformer (coordinates are listed in Section 4.1). Finally, using these matrices and the exact mass of the ${ }^{13} \mathrm{C}$ isotope (13.003355 amu) the harmonic frequencies and the respective zero-point vibrational energy (ZPVE) for each of the isotopomers were evaluated (ZPVEs are listed in Section 4.2). All the $S_{0}$ and $S_{1}$ calculations were performed at the DFT and TD-DFT levels of theory, respectively, using the CAM-B3LYP functional and 6-31G** basis set. We employ the Coulomb attenuated version of the hybrid B3LYP functional, because of the known problems of B3LYP in describing excited states with charge-transfer character. ${ }^{8}$ For all the calculations the GAMESS (US) software package ${ }^{9}$ was used.

To simulate the shape of the band origin of each conformer at $\mathrm{m} / \mathrm{z}=136.077 \mathrm{Da}$ and $\mathrm{m} / \mathrm{z}=137.080$ Da we divided all the isotopologues into two groups: those, which contain ${ }^{13} \mathrm{C}$ in the immonium fragment, and those, which contain ${ }^{13} \mathrm{C}$ in the rest part of the ion. The shapes of the band origin were evaluated as follows:

$$
\begin{gathered}
I(\tilde{v}, 136.077 D a)=(1-\alpha) \cdot I_{0}(\tilde{v})+\alpha \cdot \sum_{k} I_{0}\left(\tilde{v}-\lambda \cdot \Delta \tilde{v}_{k}\right) \\
I(\tilde{v}, 137.080 D a)=\alpha \cdot \sum_{k} I_{0}\left(\tilde{v}-\lambda \cdot \Delta \tilde{v}_{k}\right)
\end{gathered}
$$

where $\Delta \tilde{v}_{k}$ is the calculated shift of the difference between the $S_{1}$ and $S_{0}$ ZPVEs of the k-th isotopologues (from the first and the second group, respectively) relative to that of the monoisotopic species. $\alpha=0.011$ is the abundance of ${ }^{13} \mathrm{C}, \lambda=0.976$ is the scaling factor for ZPVE ${ }^{\mathbf{1 0}}$ and $I_{0}(\widetilde{v})$ is the shape of the band origin of monoisotopic species. $I_{0}(\tilde{v})$ was approximated either by a Gaussian function with FWHM $=1.77 \mathrm{~cm}^{-1}$ (see Figure S4) or by the exact shape of the band origin of component I (see Figure 1d). 


\section{Conformer A}

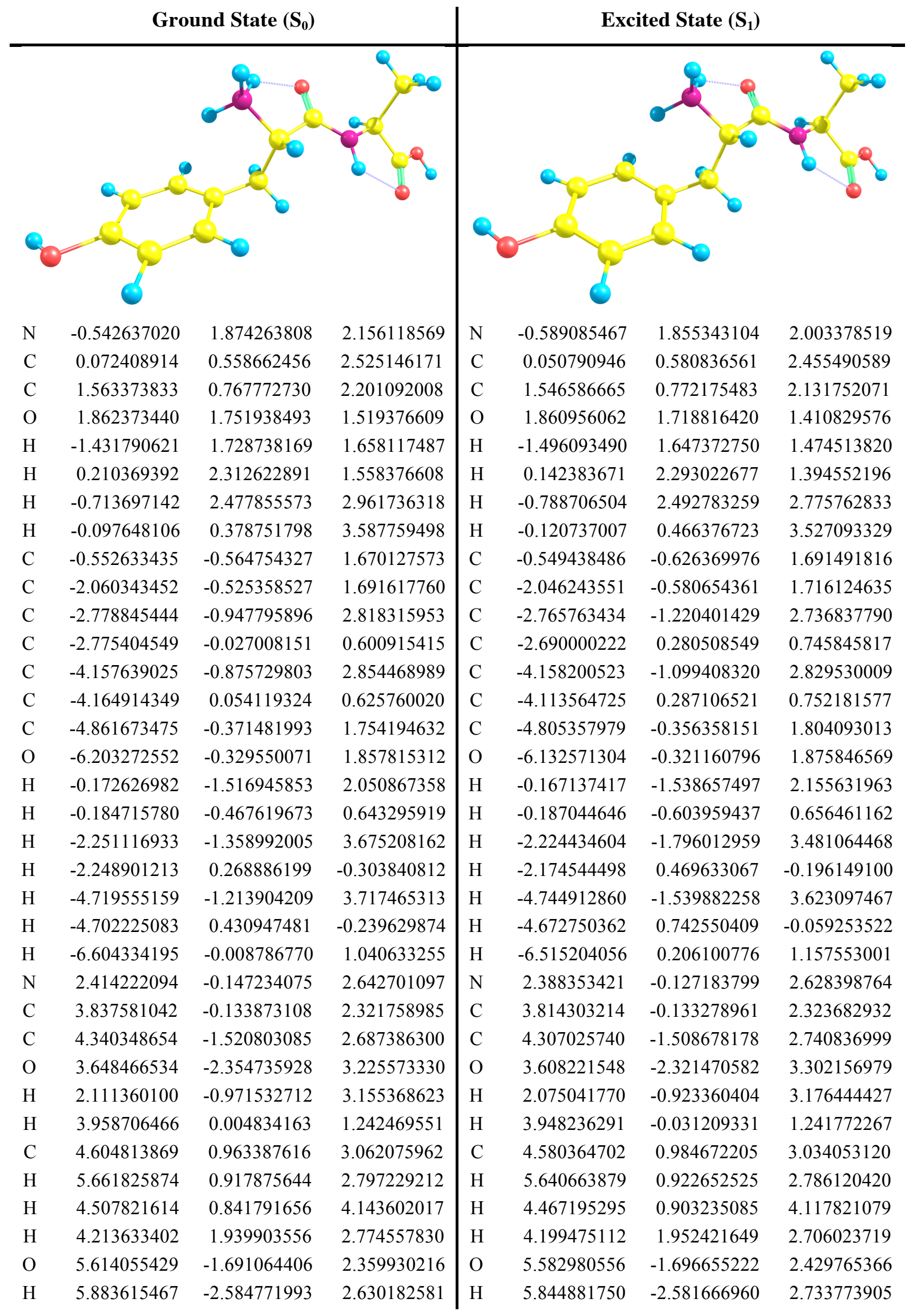




\section{Conformer B}

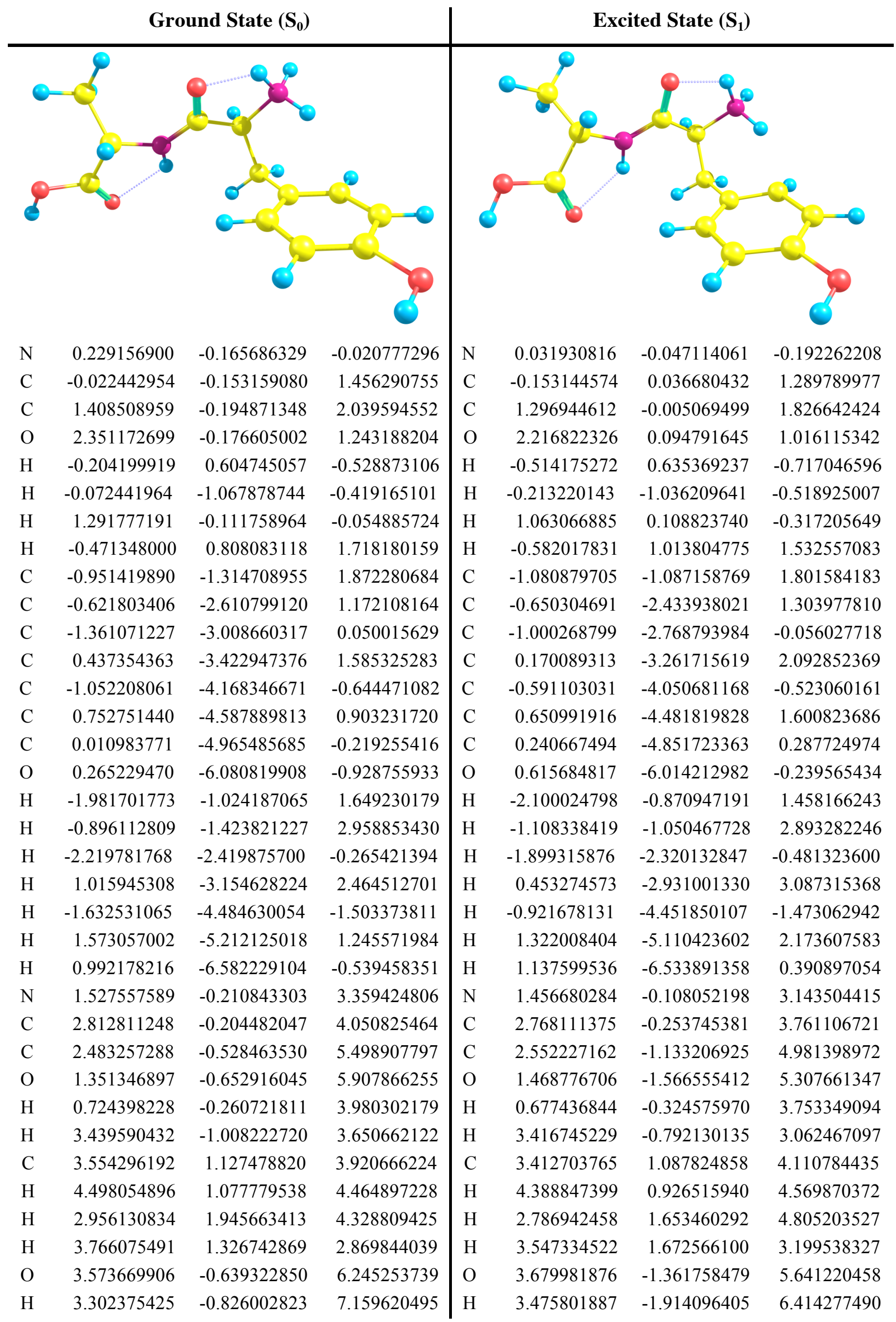




\section{Conformer C}

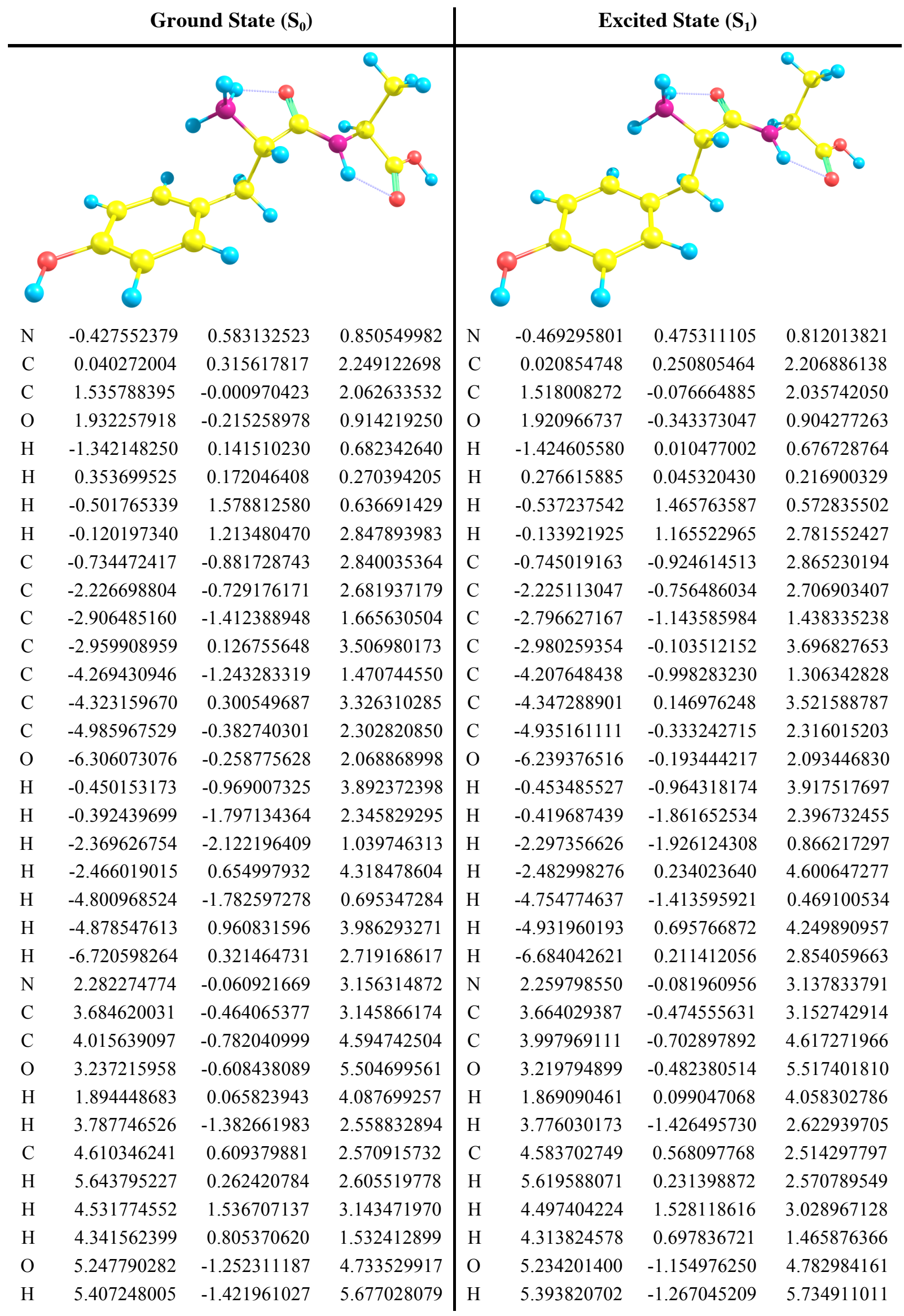




\section{Conformer D}

\begin{tabular}{|c|c|c|c|c|c|c|c|}
\hline \multicolumn{4}{|c|}{ Ground State $\left(\mathbf{S}_{0}\right)$} & \multicolumn{4}{|c|}{ Excited State $\left(S_{1}\right)$} \\
\hline & & & & & & & \\
\hline $\mathrm{N}$ & 0.633658903 & -0.647532658 & 0.058137999 & $\mathrm{~N}$ & 0.535650227 & -0.700615706 & 0.071250205 \\
\hline $\mathrm{C}$ & 0.247200875 & 0.101181627 & 1.297547255 & $\mathrm{C}$ & 0.208039276 & 0.083115921 & 1.303284244 \\
\hline $\mathrm{C}$ & 1.623659549 & 0.532739122 & 1.855473681 & $\mathrm{C}$ & 1.602628876 & 0.483273287 & 1.840932705 \\
\hline $\mathrm{O}$ & 2.602901051 & 0.389373140 & 1.118498714 & $\mathrm{O}$ & 2.578065865 & 0.287410072 & 1.116667187 \\
\hline $\mathrm{H}$ & 0.082532335 & -0.411436575 & -0.766536269 & $\mathrm{H}$ & -0.017756426 & -0.440114716 & -0.744288979 \\
\hline $\mathrm{H}$ & 0.580151620 & -1.661175285 & 0.239554877 & $\mathrm{H}$ & 0.411309440 & -1.742869354 & 0.276153127 \\
\hline $\mathrm{H}$ & 1.655647869 & -0.375810411 & -0.046880938 & $\mathrm{H}$ & 1.556721023 & -0.498185928 & -0.076039494 \\
\hline $\mathrm{H}$ & -0.285733196 & 1.004352582 & 0.987681197 & $\mathrm{H}$ & -0.318272943 & 0.995253864 & 1.005229068 \\
\hline $\mathrm{C}$ & -0.640421777 & -0.761249563 & 2.213058099 & $\mathrm{C}$ & -0.667275584 & -0.739352424 & 2.273348558 \\
\hline $\mathrm{C}$ & -0.065390668 & -2.130361158 & 2.482604927 & $\mathrm{C}$ & -0.078810686 & -2.091341323 & 2.542082783 \\
\hline $\mathrm{C}$ & 0.860253136 & -2.343634686 & 3.513127777 & $\mathrm{C}$ & 0.725810243 & -2.314000382 & 3.671501951 \\
\hline $\mathrm{C}$ & -0.434930134 & -3.221365683 & 1.691485693 & $\mathrm{C}$ & -0.271250554 & -3.099952604 & 1.521030046 \\
\hline $\mathrm{C}$ & 1.405541318 & -3.594029684 & 3.732316354 & $\mathrm{C}$ & 1.352548721 & -3.548745102 & 3.880455909 \\
\hline $\mathrm{C}$ & 0.113136037 & -4.483667122 & 1.897800294 & $\mathrm{C}$ & 0.273925909 & -4.387115819 & 1.789162812 \\
\hline $\mathrm{C}$ & 1.040089120 & -4.673375756 & 2.920403337 & $\mathrm{C}$ & 1.093401209 & -4.571021268 & 2.926478110 \\
\hline $\mathrm{O}$ & 1.613441046 & -5.861167914 & 3.190630279 & $\mathrm{O}$ & 1.659193022 & -5.746800439 & 3.181932604 \\
\hline $\mathrm{H}$ & -1.624354694 & -0.849339628 & 1.742281833 & $\mathrm{H}$ & -1.663379789 & -0.847588906 & 1.826675206 \\
\hline $\mathrm{H}$ & -0.808830163 & -0.212687361 & 3.144107844 & $\mathrm{H}$ & -0.801517707 & -0.169147557 & 3.195616751 \\
\hline $\mathrm{H}$ & 1.137313665 & -1.523929139 & 4.169798299 & $\mathrm{H}$ & 0.883935701 & -1.502396408 & 4.374967050 \\
\hline $\mathrm{H}$ & -1.202621329 & -3.103717390 & 0.928681451 & $\mathrm{H}$ & -1.163507678 & -3.033754816 & 0.895876826 \\
\hline $\mathrm{H}$ & 2.110017801 & -3.769025229 & 4.537246436 & $\mathrm{H}$ & 2.009633425 & -3.750538485 & 4.714422944 \\
\hline $\mathrm{H}$ & -0.199191418 & -5.320408224 & 1.279875944 & $\mathrm{H}$ & 0.039152187 & -5.235907983 & 1.154263867 \\
\hline $\mathrm{H}$ & 1.272454197 & -6.551545605 & 2.608818552 & $\mathrm{H}$ & 1.455450120 & -6.398318708 & 2.493277135 \\
\hline $\mathrm{N}$ & 1.664853131 & 1.066468956 & 3.068078116 & $\mathrm{~N}$ & 1.655470837 & 1.053204312 & 3.040866122 \\
\hline $\mathrm{C}$ & 2.916137297 & 1.454986769 & 3.709406812 & $\mathrm{C}$ & 2.916136956 & 1.395345496 & 3.686750088 \\
\hline $\mathrm{C}$ & 2.663150074 & 1.321948237 & 5.201605881 & $\mathrm{C}$ & 2.654892922 & 1.274473490 & 5.178545352 \\
\hline $\mathrm{O}$ & 1.594537773 & 0.990742305 & 5.665469784 & $\mathrm{O}$ & 1.578727584 & 0.967463479 & 5.642876878 \\
\hline $\mathrm{H}$ & 0.860462958 & 1.057273493 & 3.686141455 & $\mathrm{H}$ & 0.849491355 & 1.077273717 & 3.654572425 \\
\hline $\mathrm{H}$ & 3.687579422 & 0.731689206 & 3.426430195 & $\mathrm{H}$ & 3.660519603 & 0.641874618 & 3.409354607 \\
\hline $\mathrm{C}$ & 3.374266455 & 2.859240408 & 3.314776978 & $\mathrm{C}$ & 3.431805747 & 2.778901438 & 3.289942616 \\
\hline $\mathrm{H}$ & 4.306085273 & 3.104192671 & 3.825863966 & $\mathrm{H}$ & 4.369717368 & 2.989483236 & 3.805100518 \\
\hline $\mathrm{H}$ & 2.621397356 & 3.605081330 & 3.580403560 & $\mathrm{H}$ & 2.707061350 & 3.554845220 & 3.547399822 \\
\hline $\mathrm{H}$ & 3.546733016 & 2.896081622 & 2.238140424 & $\mathrm{H}$ & 3.610387279 & 2.803617803 & 2.213961212 \\
\hline $\mathrm{O}$ & 3.738596851 & 1.613588408 & 5.919805364 & $\mathrm{O}$ & 3.733379879 & 1.546448807 & 5.900703219 \\
\hline $\mathrm{H}$ & 3.514385051 & 1.521848369 & 6.861032160 & $\mathrm{H}$ & 3.501989264 & 1.462640114 & 6.840831885 \\
\hline
\end{tabular}


4.2. Zero-point vibrational energies in the ground $\left(S_{0}\right)$ and excited $\left(S_{1}\right)$ states

Conformer A

\begin{tabular}{c|c|c|c|c} 
\# of atom & ZPVE in $\mathbf{S}_{\mathbf{0}}$ (a. u.) & ZPVE in $\mathbf{S}_{\mathbf{1}}$ (a. u.) & $\Delta$ ZPVE $\left(\mathbf{c m}^{-1}\right)$ & Relative shift $\left(\mathbf{c m}^{-1}\right)$ \\
\hline \multicolumn{5}{|c}{ Monoisotopic } \\
\hline $\mathbf{5}$ & 0.295679 & 0.290539 & -1101.0 & $\mathbf{0 . 0}$ \\
\hline $\mathbf{2}$ & 0.295440 & 0.290301 & -1100.8 & $\mathbf{0 . 2}$ \\
\hline $\mathbf{3}$ & 0.295405 & 0.290265 & -1101.0 & $\mathbf{0 . 0}$ \\
\hline $\mathbf{9}$ & 0.295453 & 0.290316 & -1100.4 & $\mathbf{0 . 6}$ \\
\hline $\mathbf{1 0}$ & 0.295436 & 0.290306 & -1098.9 & $\mathbf{2 . 1}$ \\
\hline $\mathbf{1 1}$ & 0.295452 & 0.290323 & -1098.7 & $\mathbf{2 . 4}$ \\
\hline $\mathbf{1 2}$ & 0.295453 & 0.290343 & -1094.6 & $\mathbf{6 . 4}$ \\
\hline $\mathbf{1 3}$ & 0.295452 & 0.290331 & -1097.0 & $\mathbf{4 . 1}$ \\
\hline $\mathbf{1 4}$ & 0.295454 & 0.290330 & -1097.6 & $\mathbf{3 . 4}$ \\
\hline $\mathbf{1 5}$ & 0.295428 & 0.290301 & -1098.2 & $\mathbf{2 . 8}$ \\
\hline $\mathbf{2 5}$ & 0.295434 & 0.290294 & -1101.0 & $\mathbf{0 . 0}$ \\
\hline $\mathbf{2 6}$ & 0.295404 & 0.290264 & -1101.0 & $\mathbf{0 . 0}$ \\
\hline $\mathbf{3 0}$ & 0.295486 & 0.290346 & -1101.0 & $\mathbf{0 . 0}$
\end{tabular}

Conformer B

\begin{tabular}{c|c|c|c|c} 
\# of atom & ZPVE in $\mathbf{S}_{\mathbf{0}}$ (a. u.) & ZPVE in S $\mathbf{1}$ (a. u.) & $\Delta$ ZPVE $\left(\mathbf{c m}^{-\mathbf{1}}\right)$ & Relative shift $\left(\mathbf{c m}^{-\mathbf{1}}\right)$ \\
\hline \multicolumn{5}{|c}{ Monoisotopic } \\
\hline $\mathbf{5}$ & 0.296150 & 0.290734 & -1160.1 & $\mathbf{0 . 0}$ \\
\hline $\mathbf{5}$ & 0.295914 & 0.290495 & -1160.8 & $\mathbf{- 0 . 6}$ \\
\hline $\mathbf{3}$ & 0.295880 & 0.290462 & -1160.6 & $\mathbf{- 0 . 4}$ \\
\hline $\mathbf{9}$ & 0.295925 & 0.290507 & -1160.6 & $\mathbf{- 0 . 4}$ \\
\hline $\mathbf{1 0}$ & 0.295910 & 0.290500 & -1158.9 & $\mathbf{1 . 3}$ \\
\hline $\mathbf{1 1}$ & 0.295926 & 0.290536 & -1154.6 & $\mathbf{5 . 6}$ \\
\hline $\mathbf{1 2}$ & 0.295926 & 0.290517 & -1158.6 & $\mathbf{1 . 5}$ \\
\hline $\mathbf{1 3}$ & 0.295926 & 0.290522 & -1157.6 & $\mathbf{2 . 6}$ \\
\hline $\mathbf{1 4}$ & 0.295928 & 0.290529 & -1156.5 & $\mathbf{3 . 6}$ \\
\hline $\mathbf{1 5}$ & 0.295902 & 0.290496 & -1158.0 & $\mathbf{2 . 1}$ \\
\hline $\mathbf{2 5}$ & 0.295908 & 0.290489 & -1160.8 & $\mathbf{- 0 . 6}$ \\
\hline $\mathbf{2 6}$ & 0.295878 & 0.290460 & -1160.6 & $\mathbf{- 0 . 4}$ \\
\hline $\mathbf{3 0}$ & 0.295960 & 0.290541 & -1160.8 & $\mathbf{- 0 . 6}$
\end{tabular}


Conformer C

\begin{tabular}{c|c|c|c|c} 
\# of atom & ZPVE in $\mathbf{S}_{\mathbf{0}}$ (a. u.) & ZPVE in $\mathbf{S}_{\mathbf{1}}$ (a. u.) & $\Delta$ ZPVE $\left(\mathbf{c m}^{-1}\right)$ & Relative shift $\left(\mathbf{c m}^{-1}\right)$ \\
\hline \multicolumn{5}{|c}{ Monoisotopic } \\
\hline- & 0.295839 & 0.290505 & -1142.6 & $\mathbf{0 . 0}$ \\
\hline $\mathbf{5}$ & 0.295599 & 0.290267 & -1142.2 & $\mathbf{0 . 4}$ \\
\hline $\mathbf{3}$ & 0.295565 & 0.290231 & -1142.6 & $\mathbf{0 . 0}$ \\
\hline $\mathbf{9}$ & 0.295613 & 0.290282 & -1141.9 & $\mathbf{0 . 6}$ \\
\hline $\mathbf{1 0}$ & 0.295595 & 0.290271 & -1140.4 & $\mathbf{2 . 1}$ \\
\hline $\mathbf{1 1}$ & 0.295612 & 0.290308 & -1136.2 & $\mathbf{6 . 4}$ \\
\hline $\mathbf{1 2}$ & 0.295612 & 0.290289 & -1140.2 & $\mathbf{2 . 4}$ \\
\hline $\mathbf{1 3}$ & 0.295612 & 0.290293 & -1139.4 & $\mathbf{3 . 2}$ \\
\hline $\mathbf{1 4}$ & 0.295614 & 0.290300 & -1138.3 & $\mathbf{4 . 3}$ \\
\hline $\mathbf{1 5}$ & 0.295588 & 0.290267 & -1139.8 & $\mathbf{2 . 8}$ \\
\hline $\mathbf{2 5}$ & 0.295594 & 0.290260 & -1142.6 & $\mathbf{0 . 0}$ \\
\hline $\mathbf{2 6}$ & 0.295565 & 0.290230 & -1142.8 & $\mathbf{- 0 . 2}$ \\
\hline $\mathbf{3 0}$ & 0.295646 & 0.290311 & -1142.8 & $\mathbf{- 0 . 2}$
\end{tabular}

\section{Conformer D}

\begin{tabular}{c|c|c|c|c} 
\# of atom & ZPVE in S $\mathbf{0}$ (a. u.) & ZPVE in $\mathbf{S}_{\mathbf{1}}$ (a. u.) & $\Delta$ ZPVE $\left(\mathbf{c m}^{-\mathbf{1}}\right)$ & Relative shift $\left(\mathbf{c m}^{-\mathbf{1}}\right)$ \\
\hline \multicolumn{5}{|c}{ Monoisotopic } \\
\hline $\mathbf{5}$ & 0.296225 & 0.290869 & -1147.3 & $\mathbf{0 . 0}$ \\
\hline $\mathbf{5}$ & 0.295986 & 0.290630 & -1147.3 & $\mathbf{0 . 0}$ \\
\hline $\mathbf{3}$ & 0.295952 & 0.290597 & -1147.1 & $\mathbf{0 . 2}$ \\
\hline $\mathbf{9}$ & 0.295996 & 0.290642 & -1146.9 & $\mathbf{0 . 4}$ \\
\hline $\mathbf{1 0}$ & 0.295982 & 0.290636 & -1145.2 & $\mathbf{2 . 1}$ \\
\hline $\mathbf{1 1}$ & 0.295997 & 0.290652 & -1144.9 & $\mathbf{2 . 4}$ \\
\hline $\mathbf{1 2}$ & 0.295999 & 0.290672 & -1141.1 & $\mathbf{6 . 2}$ \\
\hline $\mathbf{1 3}$ & 0.295998 & 0.290661 & -1143.2 & $\mathbf{4 . 1}$ \\
\hline $\mathbf{1 4}$ & 0.296000 & 0.290660 & -1143.9 & $\mathbf{3 . 4}$ \\
\hline $\mathbf{1 5}$ & 0.295974 & 0.290631 & -1144.5 & $\mathbf{2 . 8}$ \\
\hline $\mathbf{2 5}$ & 0.295980 & 0.290623 & -1147.5 & $\mathbf{- 0 . 2}$ \\
\hline $\mathbf{2 6}$ & 0.295951 & 0.290594 & -1147.5 & $\mathbf{- 0 . 2}$ \\
\hline $\mathbf{3 0}$ & 0.296033 & 0.290676 & -1147.5 & $\mathbf{- 0 . 2}$
\end{tabular}




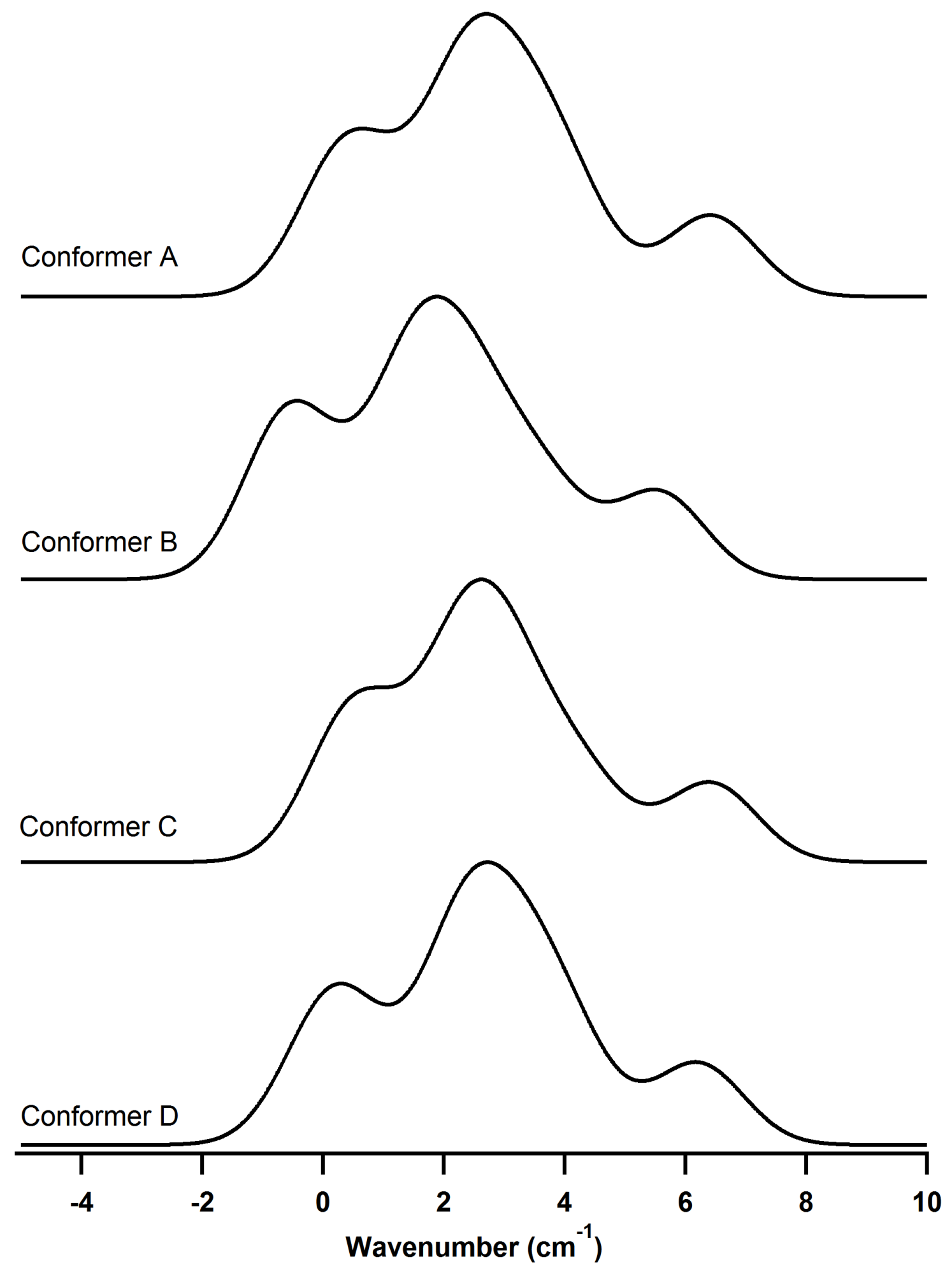

Figure S4. Simulated shape of the UV band origins at $\mathrm{m} / \mathrm{z}=137.080 \mathrm{Da}$. The wavenumber scale is relative to the band origin position of the monoisotopic conformers. 


\section{References}

(1) Boyarkin, O. V; Kopysov, V. Cryogenically Cooled Octupole Ion Trap for Spectroscopy of Biomolecular Ions. Rev. Sci. Instrum. 2014, 85, 033105.

(2) Orfanidis, S. J. Introduction to Signal Processing; Prentice Hall, 1996.

(3) Berry, M. W.; Browne, M.; Langville, A. N.; Pauca, V. P.; Plemmons, R. J. Algorithms and Applications for Approximate Nonnegative Matrix Factorization. Comput. Stat. Data Anal. 2007, 52, 155-173.

(4) Lee, D. D.; Seung, H. S. Learning the Parts of Objects by Non-Negative Matrix Factorization. Nature 1999, 401, 788-791.

(5) Owen, A. B.; Perry, P. O. Bi-Cross-Validation of the SVD and the Nonnegative Matrix Factorization. Ann. Appl. Stat. 2009, 3, 564-594.

(6) Kopysov, V.; Nagornova, N. S.; Boyarkin, O. V. Identification of Tyrosine-Phosphorylated Peptides Using Cold Ion Spectroscopy. J. Am. Chem. Soc. 2014, 136, 9288-9291.

(7) Stearns, J. A.; Guidi, M.; Boyarkin, O. V.; Rizzo, T. R. Conformation-Specific Infrared and Ultraviolet Spectroscopy of Tyrosine-Based Protonated Dipeptides. J. Chem. Phys. 2007, $127,154322$.

(8) Peach, M. J. G.; Benfield, P.; Helgaker, T.; Tozer, D. J. Excitation Energies in Density Functional Theory: An Evaluation and a Diagnostic Test. J. Chem. Phys. 2008, 128, 044118.

(9) Schmidt, M. W.; Baldridge, K. K.; Boatz, J. A.; Elbert, S. T.; Gordon, M. S.; Jensen, J. H.; Koseki, S.; Matsunaga, N.; Nguyen, K. A.; Su, S.; et al. General Atomic and Molecular Electronic Structure System. J. Comput. Chem. 1993, 14, 1347-1363.

(10) Alecu, I. M.; Zheng, J.; Zhao, Y.; Truhlar, D. G. Computational Thermochemistry: Scale Factor Databases and Scale Factors for Vibrational Frequencies Obtained from Electronic Model Chemistries. J. Chem. Theory Comput. 2010, 6, 2872-2887. 